\title{
Transparencia en entidades públicas y su relación con la implementación de sistemas de gestión y normatividad anticorrupción*
}

[Artículos de investigación]

Víctor Maldonado Nova**

Recibido: 17 de abril del 2020

Revisado: o8 de agosto del 2020

Aceptado: 31 de agosto del 2020

Cómo citar este artículo:

Maldonado Nova, V. (2020). Transparencia en entidades públicas y su relación con la implementación de sistemas de gestión y normatividad anticorrupción. Signos, Investigación en sistemas de gestión, 13(1).

https://doi.org/10.15332/24631140.6346

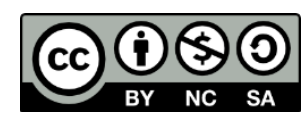

\section{Resumen}

La medición de las conductas que alteran el correcto desempeño de las entidades públicas, como la corrupción, es de vital importancia para orientar políticas y acciones concisas que mejoren la gestión financiera y

\footnotetext{
* Artículo de resultado de investigación

** Universidad Santo Tomás. Especialista en Administración y Gerencia de Sistemas de la Calidad. Administrador público. Universidad Santo Tomás, Bogotá, Colombia. Correo electrónico: vmaldonadon@gmail.com. ORCID: https://orcid.org/0000-0002-9576530X. CVLAC:

https://scienti.minciencias.gov.co/cvlac/visualizador/generarCurriculoCv.do?cod_rh $=0$ $\underline{001406671}$
} 
organizacional, y la percepción que de estas tienen los habitantes del país. Esta investigación busca establecer si los sistemas de gestión y la normatividad anticorrupción coadyuvan a que las entidades sean más transparentes y reciban mejores calificaciones en la medición del índice de transparencia nacional (ITN). Para realizar la investigación, se enviaron 40 derechos de petición a diferentes entidades nacionales a fin de caracterizarlas, establecer tendencias y determinar la correlación estadística entre el ITN, los sistemas de gestión y la normatividad anticorrupción. A partir del análisis de la información, se concluyó que la implementación de sistemas de gestión y la aplicación de la normatividad anticorrupción no inciden en el resultado del ITN de las entidades públicas estudiadas.

Palabras clave: corrupción, desempeño del gobierno, administración pública, política de gobierno, regulación.

\section{Transparency in public entities and their relationship with management systems and anti- corruption regulations implementation}

\section{Abstract}

Measuring behaviors that alter public entities proper performance, such as corruption, is of vital importance to guide policies and concise actions that improve financial and organizational management, and the perception that the country's inhabitants have of them. This research seeks to establish whether management systems and anti-corruption regulations help entities to be more transparent and receive better ratings in the measurement of the National Transparency Index (ITN in Spanish). Forty petition rights were sent to different national entities to characterize those entities, establish trends, and determine the statistical correlation between the ITN, management systems and anti-corruption regulations. From the analysis of the information, it was concluded that the implementation of management systems and the application of anti- 
corruption regulations do not affect the INT results of the public entities analyzed.

Keywords: corruption, government performance, public administration, government policy and regulation.

\section{Transparência nas entidades públicas e sua relação com a implementação de sistemas de gestão e regulamentos anticorrupção}

\section{Resumo}

A medição dos comportamentos que alteram o desempenho correto das entidades públicas, como a corrupção, é extremamente importante para nortear políticas e ações concisas que melhoram a gestão financeira e organizacional, e a percepção que a população do país tem sobre eles. Esta pesquisa procura estabelecer se os sistemas de gestão e os regulamentos anticorrupção ajudam às entidades para serem mais transparentes e receberem melhores classificações na medição do índice nacional de transparência. (ITN). Quarenta direitos de petição foram enviados a diferentes entidades nacionais para caracterizá-los, estabelecer tendências e determinar a correlação estatística entre o ITN, os sistemas de gestão e regulamentos anticorrupção. A partir da análise das informações, concluiu-se que a implementação de sistemas de gestão e a aplicação de regulamentos anticorrupção não afetam os resultados da ITN das entidades analisadas.

Palavras-chave: corrupção, desempenho do governo, administração pública, política e regulamentação do governo.

\section{Introducción}

El uso del poder para lograr un beneficio propio o corrupción es un problema que concierne a todos los gobiernos y organizaciones a nivel mundial, puesto que afecta la legitimidad y la confianza en estas organizaciones (Departamento Nacional de Planeación, 2013). Este 
fenómeno es inherente al actuar ético de las personas y cobra una gran relevancia si se considera que el recurso humano es el factor más importante con el que cuenta una empresa, organización o entidad estatal. Por tal motivo, a nivel nacional e internacional, particularmente en el sector público, los líderes gubernamentales de los países han diseñado e implementado diferentes estrategias y lineamientos para disminuir la posibilidad de ocurrencia de hechos de corrupción, mediante controles más contundentes, condenas ejemplares a políticos corruptos, mayor vinculación del ciudadano en temas de control y seguimiento del servicio público, entre otros.

En Colombia, la lucha contra esta problemática ha sido atendida por los gobiernos a través de normas (leyes y decretos de obligatorio cumplimiento para las entidades), que unifican esfuerzos para mejorar en temas de transparencia, anticorrupción y administración de los riesgos de corrupción. El objetivo es mitigar la ocurrencia de estos hechos y el impacto que podrían tener sobre la ciudadanía, la imagen de las entidades y los servidores públicos involucrados (Departamento Administrativo de la Función Pública, 2015). Así mismo, el Gobierno exige la implementación de diferentes sistemas de gestión de calidad, estipulados en la Norma Técnica de Calidad en la Gestión Pública NTC GP 1000 (República de Colombia, 2009) y en cada entidad se decide la implementación de otros sistemas de gestión como calidad ambiental, seguridad de la información, entre otros, con el fin de mejorar los procesos y satisfacer las necesidades de toda la ciudadanía.

No solo el Gobierno ha estado interesado en mejorar la gestión anticorrupción, también la sociedad civil colombiana ha manifestado la necesidad de exigir al Estado mejoras en este tema, debido al alto número de hechos de corrupción presentados en las entidades públicas. Por ello, en el año 1998 se creó la Corporación de Transparencia por Colombia 
(TpC), una organización sin ánimo de lucro llamada a liderar la lucha contra la corrupción y la transparencia.

A partir de 2002, la TpC ha planteado diferentes metodologías que permiten medir el avance de las entidades públicas en relación con la corrupción en las organizaciones colombianas, entre las cuales se encuentra el índice de transparencia nacional (ITN), que se emplea para medir e identificar el nivel de riesgo de corrupción al que están expuestas las entidades públicas del orden nacional (Corporación Transparencia por Colombia, 2017). Este índice cuenta con una metodología robusta que ha sido aplicada desde hace 15 años $\mathrm{y}$, a fin de generar controles apropiados para los riesgos de corrupción que puedan Presentarse en las entidades públicas, evalúa tres factores importantes: 1) factor de visibilidad, 2) factor de institucionalidad y 3) factor de control y sanción. En otras palabras, se evalúa la capacidad que tiene una entidad para hacer pública su gestión, dar cumplimiento a sus procesos de gestión y realizar acciones orientadas al control-sanción.

$\mathrm{Al}$ revisar la documentación existente sobre la corrupción en Colombia, incluyendo indicadores de gestión, gestión administrativa, administración pública y sistemas de gestión de calidad, se puede identificar que en Colombia no existen estudios que evidencien el aporte de la normativa anticorrupción y los sistemas de gestión de calidad a los resultados del ITN. Por tal motivo, el objetivo principal de la presente investigación fue determinar la incidencia de los sistemas de gestión y la normatividad anticorrupción en los resultados del ITN de las entidades públicas nacionales. Para esto, se plantearon diferentes metodologías orientadas a: 1) identificar y definir el aporte de la normatividad y los sistemas de gestión al resultado del ITN; 2) caracterizar las entidades públicas de acuerdo con los resultados del ITN durante 2014 y 2016, y 3) establecer y analizar la correlación de los resultados del ITN frente a la 
implementación de sistemas de gestión y aplicación de la normatividad anticorrupción en las entidades.

\section{Metodología}

La investigación se realizó en 40 entidades públicas del orden nacional medidas por el ITN en dos momentos: 2014 y 2016. El resultado del ITN se relacionó con los factores que lo componen, la implementación de sistemas de gestión y la aplicación de la normatividad anticorrupción. Esta exploración cumple criterios inherentes a la investigación cuantitativa, como son: debe existir relación entre variables, debe formularse en forma de pregunta y la pregunta planteada debe poder probarse (HernándezSampieri et ál., 2014). Adicionalmente, se empleó un diseño no experimental transversal, que, según Hernández-Sampieri et ál. (2014), busca recolectar datos en un momento determinado y su intención es describir las variables y su interrelación.

Con base en lo anterior, se estructuró el modelo conceptual (figura 1) en el cual se presentan y desarrollan las principales variables de la investigación. Se evidencia que los sistemas de gestión y la normatividad anticorrupción afectan los factores de visibilidad, institucionalidad y control-sanción del índice de transparencia de entidades públicas nacionales y cómo estas repercuten en la transparencia de la organización, con énfasis en la relación existente entre los conceptos de sistemas de gestión y la transparencia de la organización. 
Figura 1. Modelo conceptual

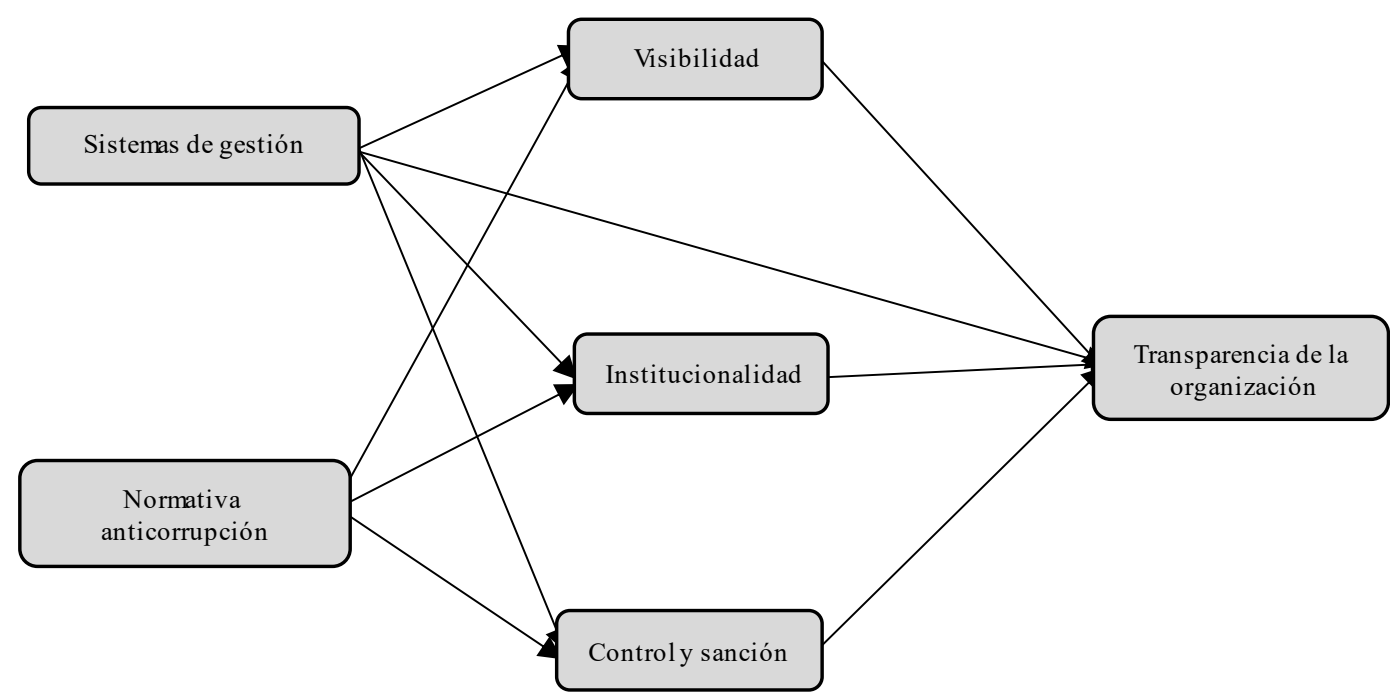

Fuente: elaboración propia.

De acuerdo con el modelo conceptual, se trazaron diez hipótesis derivadas de la interrelación de los conceptos (tabla 1).

Tabla 1. Hipótesis para comprobación

\begin{tabular}{|c|c|}
\hline Modelo conceptual & Hipótesis \\
\hline \multirow{6}{*}{$\begin{array}{l}\text { Los sistemas de gestión y la } \\
\text { normatividad anticorrupción } \\
\text { afectan la visibilidad, la } \\
\text { institucionalidad y el control- } \\
\text { sanción. }\end{array}$} & $\begin{array}{l}\text { H1. Los sistemas de gestión afectan positivamente la } \\
\text { visibilidad. }\end{array}$ \\
\hline & $\begin{array}{l}\text { H3. Los sistemas de gestión afectan positivamente la } \\
\text { institucionalidad. }\end{array}$ \\
\hline & $\begin{array}{l}\text { H4. Los sistemas de gestión afectan positivamente el } \\
\text { control-sanción. }\end{array}$ \\
\hline & $\begin{array}{l}\text { H5. La normatividad anticorrupción afecta } \\
\text { positivamente la visibilidad. }\end{array}$ \\
\hline & $\begin{array}{l}\text { H6. La normatividad anticorrupción afecta } \\
\text { positivamente la institucionalidad. }\end{array}$ \\
\hline & $\begin{array}{l}\text { H7. La normatividad anticorrupción afecta } \\
\text { positivamente el control-sanción. }\end{array}$ \\
\hline \multirow{3}{*}{$\begin{array}{l}\text { La visibilidad, la } \\
\text { institucionalidad y el control- } \\
\text { sanción afectan la } \\
\text { transparencia de la } \\
\text { organización. }\end{array}$} & $\begin{array}{l}\text { H8. La visibilidad se interrelaciona con la transparencia } \\
\text { de la organización. }\end{array}$ \\
\hline & $\begin{array}{l}\text { H9. La institucionalidad se interrelaciona con la } \\
\text { transparencia de la organización. }\end{array}$ \\
\hline & $\begin{array}{l}\text { H10. El control y la sanción se interrelacionan con la } \\
\text { transparencia de la organización. }\end{array}$ \\
\hline
\end{tabular}

SIGNOS, Investigación en Sistemas de Gestión

ISSN: 2145-1389 | e-ISSN: 2463-1140 | DOI: https://doi.org/10.15332/24631140

Vol. 13 N.o 1 | enero-junio de 2021 


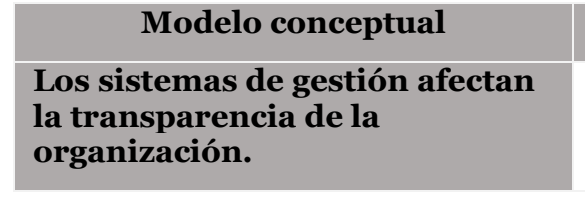

Fuente: elaboración propia.

\section{Hipótesis}

H2. Los sistemas de gestión aportan positivamente a la transparencia de la organización.

Esta metodología se desarrolló en tres fases: 1) diseño de un método para determinar el aporte de la normatividad legal y técnica en el ITN, 2) método para la caracterización de las entidades y 3) método de correlación estadística de las variables y su interpretación. Con el fin de recopilar la información necesaria, se diseñaron los instrumentos que se describen a continuación.

\section{Instrumentos de recolección de datos}

La identificación de aportes de la normatividad y el análisis estadístico requirieron el diseño de una matriz de información sobre la normatividad anticorrupción y técnica. En la primera sección de esta matriz, se incluyó un listado de normas legales y normas técnicas identificadas previamente en el marco legal de la investigación. En la segunda sección, se analizó el aporte de la normativa en cada uno de los factores del ITN y la calificación de la relación de acuerdo con este análisis.

Posteriormente, se diseñó la matriz de captura de información para la caracterización de entidades, que contiene el año en el cual se realizó la medición del ITN, los resultados del índice y subíndices (factor), algunos factores comunes de las entidades nacionales, las normas técnicas comunes que aplican y la normatividad legal resultado de la revisión realizada.

\section{Método para determinar el aporte de la normatividad al ITN}

Para determinar el aporte de la normatividad legal y técnica, se analizó el documento metodológico del ITN y se identificó que los factores del índice 
se evalúan a través de indicadores y subindicadores que permiten determinar la calificación de los factores y el ITN de las entidades. Así mismo, se efectuó un análisis exhaustivo de las normas legales (leyes y decretos) identificadas en el marco legal de la investigación, revisando sistemáticamente los lineamientos y orientaciones que el Gobierno nacional asigna a las entidades como deberes para fortalecer su transparencia, mecanismos anticorrupción, atención al ciudadano, rendición de cuentas, comportamiento ético de servidores públicos, entre otros. En el caso de los sistemas de gestión identificados, se realizó el análisis de los numerales y su aporte al fortalecimiento de los procesos de las entidades para garantizar la calidad de sus servicios o productos entregados a los ciudadanos, así como la calidad de sus procesos internos y externos.

Estas revisiones se enfocaron en identificar palabras comunes, contextos similares y relaciones entre los factores, los indicadores, los subindicadores y la normativa, para así determinar el aporte y diligenciar la matriz de recolección y análisis. Para determinar la calificación de la relación que existe entre la norma y cada uno de los factores del ITN, se diseñó una escala (tabla 2) en la que se parametrizan los niveles de relación (alta, media, baja, nula) de acuerdo con los criterios identificados en el análisis de la normativa y el documento metodológico ITN.

Tabla 2. Criterios de relación entre la norma y los factores del ITN

\begin{tabular}{|l|l|}
\hline \multicolumn{1}{|c|}{ Relación } & \multicolumn{1}{c}{ Criterio } \\
\hline Alta & $\begin{array}{l}\text { Aporta directamente a un indicador o subindicador } \\
\text { Análisis de relación (norma-factor) positivo }\end{array}$ \\
\hline Media & $\begin{array}{l}\text { Aporta indirecta o parcialmente a un indicador o subindicador } \\
\text { Análisis de relación (norma-factor) positivo }\end{array}$ \\
\hline Baja & $\begin{array}{l}\text { Aporta indirecta o parcialmente a un indicador o subindicador } \\
\text { Análisis de relación (norma-factor) negativo }\end{array}$ \\
\hline Nula & No hay aporte a los indicadores \\
\hline
\end{tabular}


Fuente: elaboración propia.

\section{Método para la caracterización de entidades}

Con el fin de caracterizar las entidades públicas del orden nacional medidas por el ITN, se describieron los datos obtenidos realizando cruces de variables en Microsoft Excel 2018. Adicionalmente, se realizó estadística descriptiva, entendida como aquella que permite describir y analizar un grupo específico sin generar inferencias generalizables (Spiegel y Stephens, 2009). Para tal efecto, en el software SPSS y Microsoft Excel 2018 se ejecutaron las fórmulas para determinar medidas de tendencia central como media, mediana y moda, así como medidas de dispersión como desviación estándar, varianza y rango, y se realizaron cruces entre las variables de la investigación generando representaciones gráficas (histogramas) y tablas de frecuencias.

\section{Método para determinar la correlación estadística y su interpretación}

Para procesar los datos, se realizó la prueba estadística no paramétrica llamada correlación de rango de Spearman (Spiegel y Stephens, 2009), debido a que las variables escogidas para esta investigación son tanto discretas como ordinales. Se empleó la siguiente fórmula de cálculo:

$$
r_{s=1-\frac{6 \sum d^{2}}{n\left(n^{2}-1\right)}}
$$

Donde $d$ es la diferencia entre el rango de las variables $x$ y $y$, y $n$ es el número de datos obtenidos en la muestra realizada. El resultado de la aplicación de esta fórmula fue interpretado de acuerdo con la tabla 3. 
Tabla 3. Interpretación del coeficiente de Spearman

\begin{tabular}{|c|c|}
\hline Correlación perfecta cuando $r=1$ & $\mathbf{r}-\mathbf{1}$ \\
\hline $\begin{array}{l}\text { Correlación excelente cuando r es mayor de } 0,90 \text { y menor } \\
\text { de } 1\end{array}$ & $(-1<\mathrm{r}<-0,90)$ \\
\hline $\begin{array}{l}\text { Correlación aceptable cuando r se encuentra entre } 0,80 \text { y } \\
0,90\end{array}$ & $(-0,90<\mathrm{r}<-0,80)$ \\
\hline $\begin{array}{l}\text { Correlación regular cuando r se encuentra entre } 0,60 \text { y } \\
0,80\end{array}$ & $(-0,80<r<-0,60)$ \\
\hline $\begin{array}{l}\text { Correlación mínima cuando r se encuentra entre } 0,30 \text { y } \\
0,60\end{array}$ & $(-0,60<r<-0,30)$ \\
\hline No hay correlación para $r$ menor de o,30 y mayor de o & $(-0,30<\mathrm{r}<-0)$ \\
\hline
\end{tabular}

Fuente: Martínez (2012).

\section{Recolección de datos}

La recolección de los datos necesarios para el desarrollo de los métodos planteados se realizó empleando distintas técnicas de recolección que no en todos los casos fueron eficaces debido a la naturaleza de los datos y las organizaciones.

En primer lugar, la normatividad anticorrupción y los sistemas de gestión aplicables a las entidades públicas del orden nacional se obtuvieron del marco legal de la investigación y la normatividad identificada en el documento metodológico del ITN. Es importante resaltar que el marco legal fue planteado con base en la revisión y el análisis preliminar de las leyes y los decretos que tuvieran una posible incidencia en temas anticorrupción del Estado colombiano, y que se encontraron bases de datos jurídicas, páginas web institucionales y textos jurídicos.

Posteriormente, se realizó la revisión de las páginas web institucionales de las 40 entidades que componen la muestra, con el fin de identificar las políticas, las herramientas y los controles establecidos por las normas legales como la divulgación de la información pública, la gestión administrativa y la gestión presupuestal y financiera, así como los trámites 
sobre servicio al ciudadano, políticas y medidas anticorrupción, gestión de la planeación, políticas de comportamiento ético y organizacional, gestión de la contratación, gestión de talento humano, sistema de pqrs, rendición de cuentas a la ciudadanía, control social, control institucional, control interno disciplinario y de gestión, y gobierno en línea.

En paralelo, se verificaron las bases de datos publicadas en las páginas web del Departamento Nacional de Planeación (DNP), el Departamento Administrativo de la Función Pública (DAFP) y el Departamento Administrativo Nacional de Estadística (DANE), entidades cabeza de sector que realizan seguimiento a la implementación de políticas y disposiciones del Gobierno nacional en temas de planeación y empleo público, con el fin de hallar información con respecto a la implementación de los sistemas de gestión, la cantidad de contratos y los funcionarios que componen la planta de las entidades.

Debido a que esta investigación se realizó en un tiempo reciente, en la exploración y revisión de las páginas web institucionales no fue posible determinar que en 2014 y 2016 los factores anteriormente nombrados se aplicaron en las entidades, puesto que estas páginas se actualizaron y las versiones anteriores se sobrescribieron. Asimismo, como resultado de la revisión de bases de datos, se encontró que no se tiene información específica publicada; la información disponible corresponde a datos agregados que no aportan al diligenciamiento del formato de recolección de información.

Considerando que las páginas web institucionales y las bases de datos no fueron una fuente de información suficiente para completar la recopilación de la información, se enviaron derechos de petición a 60 entidades del listado de 75 entidades que fueron medidas por el ITN en los dos periodos de tiempo, solicitando la solución de 14 preguntas. Es pertinente aclarar que, si bien la muestra es de 40 entidades, fue necesario enviar 20 
derechos de petición adicionales previendo que algunas entidades no los contestarían.

\section{Análisis estadístico}

El análisis estadístico se realizó en tres momentos. Primero, se interpretaron y analizaron los resultados del cruce de las variables, las gráficas y las tablas de medidas de tendencia central y dispersión, para así caracterizar las entidades de acuerdo con la información disponible. Luego, se aplicó la prueba estadística de Spearman, en la cual se utilizó la fórmula a los datos recolectados. Para este análisis, las variables cuantitativas discretas correspondieron a la calificación del ITN y sus factores, y se expresaron en valores absolutos. La aplicación de las normas legales y técnicas $(\mathrm{O}=$ no aplica y $1=$ aplica) correspondieron a variables ordinales. Para el efecto, se procesó la información aplicando el coeficiente de correlación rho de Spearman mediante el software SPSS Stadistics, versión 25. Por último, con base en los datos disponibles y de acuerdo con el método empleado para el cálculo de la muestra (probabilístico), se realizó un análisis de esta y se generaron deducciones con respecto a la población.

\section{Resultados y discusión}

La recolección, análisis y procesamiento de los datos se presentan de acuerdo con los métodos planteados para el desarrollo de la investigación. En primer lugar, se identificaron los sistemas de gestión y la normatividad anticorrupción aplicable a las entidades públicas del orden nacional. Así mismo, se definió el aporte de estos al resultado del ITN. En segundo lugar, se caracterizaron las entidades de acuerdo con los resultados del ITN 2014-2016. En tercer lugar, se analizaron los resultados del ITN 
respecto a la implementación de sistemas de gestión y aplicación de la normatividad anticorrupción en las entidades.

\section{Sistemas de gestión y normatividad anticorrupción en las entidades públicas nacionales}

Como resultado del método para determinar el aporte de la normatividad al ITN, las leyes, los decretos y las normas técnicas se calificaron según los factores visibilidad, institucionalidad y control-sanción (tabla 4).

Tabla 4. Normatividad aportante a los factores del ITN

\begin{tabular}{|c|c|c|c|c|c|}
\hline \multicolumn{3}{|c|}{ Referencia legal o norma técnica } & \multicolumn{3}{|c|}{ Índice de transparencia nacional } \\
\hline \multirow[t]{2}{*}{$\begin{array}{l}\text { Jerarquía } \\
\text { normativa }\end{array}$} & \multirow[t]{2}{*}{ Número } & \multirow[t]{2}{*}{$\begin{array}{c}\text { Año de } \\
\text { expedición }\end{array}$} & $\begin{array}{c}\text { Factor de } \\
\text { visibilidad }\end{array}$ & $\begin{array}{c}\text { Factor de } \\
\text { institucionalidad }\end{array}$ & $\begin{array}{l}\text { Factor de } \\
\text { control- } \\
\text { sanción }\end{array}$ \\
\hline & & & Relación & Relación & Relación \\
\hline Ley & 80 & 1993 & Alta & Alta & Alta \\
\hline Ley & 872 & 2003 & Alta & Alta & Alta \\
\hline Ley & 1150 & 2007 & Alta & Alta & Alta \\
\hline Ley & 1474 & 2011 & Alta & Alta & Alta \\
\hline Ley & 1712 & 2014 & Alta & Alta & Alta \\
\hline Decreto & 019 & 2012 & Alta & Alta & Alta \\
\hline Decreto & 103 & 2015 & Alta & Alta & Alta \\
\hline NTC ISO & 14001 & 2004 & Alta & Alta & Alta \\
\hline NTC ISO & 9001 & 2008 & Alta & Alta & Alta \\
\hline NTC-GP & 1000 & 2009 & Alta & Alta & Alta \\
\hline NTC ISO/IEC & 27001 & 2013 & Alta & Alta & Alta \\
\hline NTC-OHSAS & 18001 & 2007 & Alta & Alta & Alta \\
\hline
\end{tabular}

Fuente: elaboración propia.

Para la investigación, se descartó la participación o selección de la Ley 872 de 2003, por la cual se crea el sistema de gestión de la calidad en la rama ejecutiva del poder público y en otras entidades prestadoras de servicios, y del Decreto 103 de 2015, por el cual se reglamenta parcialmente la Ley 1712 de 2014 y se dictan otras disposiciones, ya que la norma NTC GP 
1000 de 2009 reúne y especifica todos los requisitos de calidad aplicables a las entidades públicas y es una herramienta de gestión que permite diseñar, ejecutar, evaluar y controlar el desempeño de las entidades públicas. El decreto reglamenta parcialmente la Ley 1712 de 2014, que cobija la transparencia y el derecho de acceso a la información pública nacional.

\section{Caracterización de las entidades nacionales según el ITN}

\section{4-2016}

En esta sección, se realizó una descripción particular para cada entidad, en la que se identificó 1) objeto, 2) variación que hubo del ITN, 3) variación de los factores entre la vigencia 2014 y 2016, 4) variación porcentual presupuestal y 5) variación de la contratación. Además, se procesó la estadística descriptiva señalada en el método para la caracterización de entidades y se obtuvieron las medidas de tendencia central y dispersión para las vigencias analizadas (figura 2 y 3 ).

Figura 2. Dispersión de entidades con respecto al ITN 2014

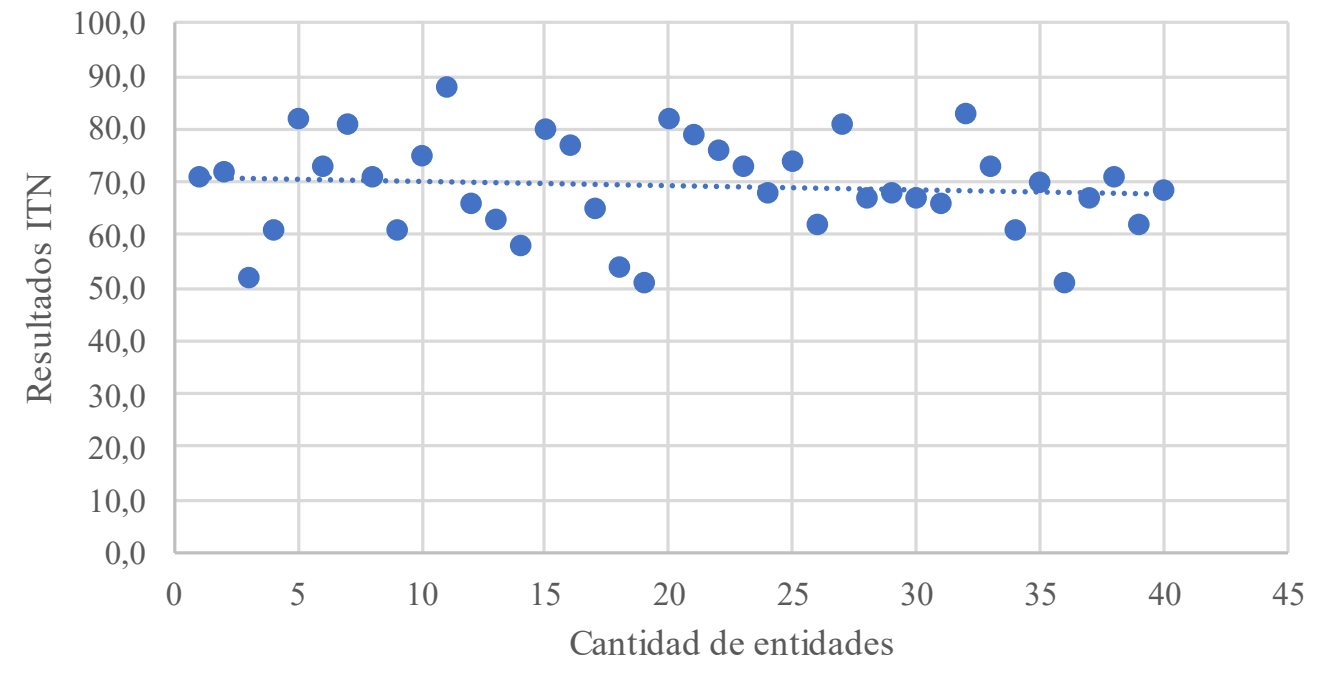

Fuente: elaboración propia. 
Figura 3. Dispersión de entidades con respecto al ITN 2016

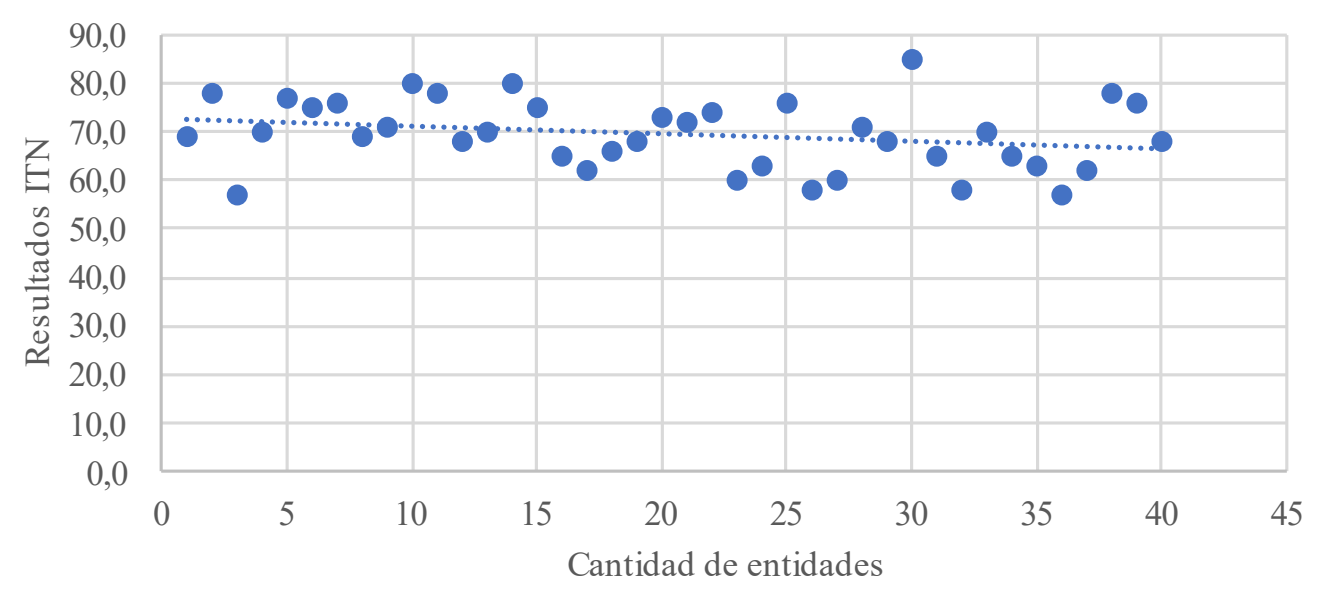

Fuente: elaboración propia.

Se puede observar en las gráficas que las medidas de tendencia central y de dispersión han mejorado los puntajes del ITN de las entidades y han disminuido su dispersión con respecto a la media. Si bien el cambio no es significativo, la tendencia de las entidades de la muestra (grupo) cuenta con un avance positivo de acuerdo con los datos recopilados por la Corporación Transparencia por Colombia (TpC).

\section{Correlación de ITN, sistemas de gestión y normatividad anticorrupción}

Se tabularon los resultados del cálculo de acuerdo con el modelo conceptual, las hipótesis planteadas y las variables involucradas. Así mismo, se realizó la interpretación de acuerdo con la escala presentada en la tabla 3. Los resultados se muestran en la tabla 5 . 
Tabla 5. Comprobación de hipótesis del modelo conceptual "Los sistemas de gestión y la normatividad anticorrupción afectan la visibilidad, institucionalidad y el control-sanción"

\begin{tabular}{|c|c|c|c|c|c|}
\hline $\begin{array}{c}\text { Modelo } \\
\text { conceptual }\end{array}$ & Hipótesis & Variable 1 & Variable 2 & $\begin{array}{l}\text { Coeficien } \\
\text { te de } \\
\text { correlaci } \\
\quad \text { ón }\end{array}$ & Resultado \\
\hline \multirow{19}{*}{$\begin{array}{l}\text { Los sistemas } \\
\text { de gestión y la } \\
\text { normatividad } \\
\text { anticorrupció } \\
\text { n afectan la } \\
\text { visibilidad, la } \\
\text { institucionalid } \\
\text { ad y el control- } \\
\text { sanción. }\end{array}$} & \multirow{5}{*}{$\begin{array}{l}\text { H1. Los } \\
\text { sistemas de } \\
\text { gestión afectan } \\
\text { positivamente } \\
\text { la visibilidad. }\end{array}$} & \multirow[t]{5}{*}{$\begin{array}{l}\text { Factor de } \\
\text { visibilidad }\end{array}$} & $\begin{array}{l}\text { ISO } \\
\text { 9001:2008 }\end{array}$ & 0,194 & No hay correlación \\
\hline & & & $\begin{array}{l}\text { ISO } \\
\text { 14001:2004 }\end{array}$ & $-0,015$ & $\begin{array}{l}\text { No hay correlación } \\
(-)\end{array}$ \\
\hline & & & $\begin{array}{l}\text { NTC GP } \\
1000: 2009\end{array}$ & 0,234 & No hay correlación \\
\hline & & & $\begin{array}{l}\text { OHSAS } \\
\text { 18001:2007 }\end{array}$ & 0,002 & No hay correlación \\
\hline & & & $\begin{array}{l}\text { ISO } \\
\text { 27001:2013 }\end{array}$ & $-0,058$ & $\begin{array}{l}\text { No hay correlación } \\
(-)\end{array}$ \\
\hline & \multirow{5}{*}{$\begin{array}{l}\text { H3. Los } \\
\text { sistemas de } \\
\text { gestión afectan } \\
\text { positivamente } \\
\text { la } \\
\text { institucionalid } \\
\text { ad. }\end{array}$} & \multirow[t]{5}{*}{$\begin{array}{l}\text { Factor de } \\
\text { institucionalid } \\
\text { ad }\end{array}$} & $\begin{array}{l}\text { ISO } \\
\text { 9001:2008 }\end{array}$ & 0,074 & No hay correlación \\
\hline & & & $\begin{array}{l}\text { ISO } \\
\text { 14001:2004 }\end{array}$ & $-0,037$ & $\begin{array}{l}\text { No hay correlación } \\
(-)\end{array}$ \\
\hline & & & $\begin{array}{l}\text { NTC GP } \\
\text { 1000:2009 }\end{array}$ & 0,030 & No hay correlación \\
\hline & & & $\begin{array}{l}\text { OHSAS } \\
\text { 18001:2007 }\end{array}$ & $-0,073$ & $\begin{array}{l}\text { No hay correlación } \\
(-)\end{array}$ \\
\hline & & & $\begin{array}{l}\text { ISO } \\
\text { 27001:2013 }\end{array}$ & 0,045 & No hay correlación \\
\hline & \multirow{5}{*}{$\begin{array}{l}\text { H4. Los } \\
\text { sistemas de } \\
\text { gestión afectan } \\
\text { positivamente } \\
\text { el control- } \\
\text { sanción. }\end{array}$} & \multirow{5}{*}{$\begin{array}{l}\text { Factor de } \\
\text { control- } \\
\text { sanción }\end{array}$} & $\begin{array}{l}\text { ISO } \\
9001: 2008\end{array}$ & 0,059 & No hay correlación \\
\hline & & & $\begin{array}{l}\text { ISO } \\
\text { 14001:2004 }\end{array}$ & $-0,076$ & $\begin{array}{l}\text { No hay correlación } \\
(-)\end{array}$ \\
\hline & & & $\begin{array}{l}\text { NTC GP } \\
\text { 1000:2009 }\end{array}$ & 0,101 & No hay correlación \\
\hline & & & $\begin{array}{l}\text { OHSAS } \\
\text { 18001:2007 }\end{array}$ & $-0,010$ & $\begin{array}{l}\text { No hay correlación } \\
(-)\end{array}$ \\
\hline & & & $\begin{array}{l}\text { ISO } \\
\text { 27001:2013 }\end{array}$ & 0,092 & No hay correlación \\
\hline & \multirow{4}{*}{$\begin{array}{l}\mathrm{H} 5 . \mathrm{La} \\
\text { normatividad } \\
\text { anticorrupción } \\
\text { afecta } \\
\text { positivamente } \\
\text { la visibilidad. }\end{array}$} & $\begin{array}{l}\text { Ley } 80 \text { de } \\
1993\end{array}$ & \multirow[t]{4}{*}{$\begin{array}{l}\text { Factor de } \\
\text { visibilidad }\end{array}$} & $-0,114$ & $\begin{array}{l}\text { No hay correlación } \\
(-)\end{array}$ \\
\hline & & $\begin{array}{l}\text { Ley } 1150 \text { de } \\
2007\end{array}$ & & $-0,114$ & $\begin{array}{l}\text { No hay correlación } \\
(-)\end{array}$ \\
\hline & & $\begin{array}{l}\text { Ley } 1474 \text { de } \\
2011\end{array}$ & & $-0,114$ & $\begin{array}{l}\text { No hay correlación } \\
(-)\end{array}$ \\
\hline & & $\begin{array}{l}\text { Ley } 1712 \text { de } \\
2014\end{array}$ & & $-0,033$ & $\begin{array}{l}\text { No hay correlación } \\
(-)\end{array}$ \\
\hline
\end{tabular}

SIGNOS, Investigación en Sistemas de Gestión

ISSN: 2145-1389 | e-ISSN: 2463-1140 | DOI: https://doi.org/10.15332/24631140

Vol. 13 N. ${ }^{0} 1$ | enero-junio de 2021 


\begin{tabular}{|c|c|c|c|c|c|}
\hline $\begin{array}{l}\text { Modelo } \\
\text { conceptual }\end{array}$ & Hipótesis & Variable 1 & Variable 2 & $\begin{array}{l}\text { Coeficien } \\
\text { te de } \\
\text { correlaci } \\
\quad \text { ón }\end{array}$ & Resultado \\
\hline & & $\begin{array}{l}\text { Decreto } 19 \text { de } \\
2012\end{array}$ & & $-0,114$ & $\begin{array}{l}\text { No hay correlación } \\
(-)\end{array}$ \\
\hline & \multirow{5}{*}{$\begin{array}{l}\text { H6. La } \\
\text { normatividad } \\
\text { anticorrupción } \\
\text { afecta } \\
\text { positivamente } \\
\text { la } \\
\text { institucionalid } \\
\text { ad. }\end{array}$} & $\begin{array}{l}\text { Ley } 80 \text { de } \\
1993\end{array}$ & \multirow{5}{*}{$\begin{array}{l}\text { Factor de } \\
\text { institucionalid } \\
\text { ad }\end{array}$} & $-0,055$ & $\begin{array}{l}\text { No hay correlación } \\
(-)\end{array}$ \\
\hline & & $\begin{array}{l}\text { Ley } 1150 \mathrm{de} \\
2007\end{array}$ & & $-0,055$ & $\begin{array}{l}\text { No hay correlación } \\
(-)\end{array}$ \\
\hline & & $\begin{array}{l}\text { Ley } 1474 \text { de } \\
2011\end{array}$ & & $-0,055$ & $\begin{array}{l}\text { No hay correlación } \\
(-)\end{array}$ \\
\hline & & $\begin{array}{l}\text { Ley } 1712 \text { de } \\
2014\end{array}$ & & $-0,124$ & $\begin{array}{l}\text { No hay correlación } \\
(-)\end{array}$ \\
\hline & & $\begin{array}{l}\text { Decreto } 19 \text { de } \\
2012\end{array}$ & & $-0,055$ & $\begin{array}{l}\text { No hay correlación } \\
(-)\end{array}$ \\
\hline & \multirow{5}{*}{$\begin{array}{l}\text { H7. La } \\
\text { normatividad } \\
\text { anticorrupción } \\
\text { afecta } \\
\text { positivamente } \\
\text { el control- } \\
\text { sanción. }\end{array}$} & $\begin{array}{l}\text { Ley } 80 \text { de } \\
1993\end{array}$ & \multirow{5}{*}{$\begin{array}{l}\text { Factor de } \\
\text { control y } \\
\text { sanción }\end{array}$} & $-0,191$ & $\begin{array}{l}\text { No hay correlación } \\
(-)\end{array}$ \\
\hline & & $\begin{array}{l}\text { Ley } 1150 \mathrm{de} \\
2007\end{array}$ & & $-0,191$ & $\begin{array}{l}\text { No hay correlación } \\
(-)\end{array}$ \\
\hline & & $\begin{array}{l}\text { Ley } 1474 \text { de } \\
2011\end{array}$ & & $-0,191$ & $\begin{array}{l}\text { No hay correlación } \\
(-)\end{array}$ \\
\hline & & $\begin{array}{l}\text { Ley } 1712 \text { de } \\
2014\end{array}$ & & $-0,150$ & $\begin{array}{l}\text { No hay correlación } \\
(-)\end{array}$ \\
\hline & & $\begin{array}{l}\text { Decreto } 19 \text { de } \\
2012\end{array}$ & & $-0,191$ & $\begin{array}{l}\text { No hay correlación } \\
(-)\end{array}$ \\
\hline
\end{tabular}

Fuente: elaboración propia.

Tras calcular el rho de Spearman, las hipótesis 1, 3, 4, 5, 6 y 7 fueron rechazadas debido a la falta de correlación entre las variables. El análisis de correspondencia entre las variables de la columna Variable 1 y las variables de la columna Variable 2 indica que no hay correlación en ningún cruce de variables, teniendo en cuenta que los resultados obtenidos oscilan entre -0,191 y 0,234.

Por otra parte, la correlación entre las variables visibilidad y resultado de ITN (tabla 6) evidencia un rho de o,611, lo que indica una correlación regular. Así mismo, la correlación entre las variables factor de institucionalidad, factor de control-sanción y resultado de ITN tienen 
coeficientes de 0,823 y 0,833 , respectivamente, lo que señala una correlación aceptable.

Considerando los coeficientes, la hipótesis 8 (la visibilidad se interrelaciona con la transparencia de la organización) se aprueba debido a la correlación entre las variables. Sin embargo, la correlación existente entre estas variables se explica en que los resultados obtenidos en cada uno de los factores son componentes del resultado general del ITN, cada una con una calificación independiente que al ser ponderada aporta en un porcentaje al índice.

Tabla 6. Comprobación de hipótesis del modelo conceptual "La visibilidad, institucionalidad y control-sanción afectan la transparencia de la organización"

\begin{tabular}{|c|c|c|c|c|c|}
\hline $\begin{array}{l}\text { Modelo } \\
\text { conceptual }\end{array}$ & Hipótesis & Variable 1 & $\begin{array}{l}\text { Variabl } \\
\text { e } 2\end{array}$ & $\begin{array}{c}\text { Coeficien } \\
\text { te de } \\
\text { correlaci } \\
\text { ón }\end{array}$ & Resultado \\
\hline \multirow[t]{3}{*}{$\begin{array}{l}\text { La visibilidad, } \\
\text { institucionali } \\
\text { dad y control- } \\
\text { sanción } \\
\text { afectan la } \\
\text { transparencia } \\
\text { de la } \\
\text { organización. }\end{array}$} & $\begin{array}{l}\text { H8. La } \\
\text { visibilidad se } \\
\text { interrelacion } \\
\text { a con la } \\
\text { transparencia } \\
\text { de la } \\
\text { organización. }\end{array}$ & $\begin{array}{l}\text { Factor de } \\
\text { visibilidad }\end{array}$ & $\begin{array}{l}\text { Resultad } \\
\text { o del ITN } \\
\text { entidades } \\
\text { orden } \\
\text { nacional }\end{array}$ & 0,611 & $\begin{array}{l}\text { Correlación } \\
\text { regular }\end{array}$ \\
\hline & $\begin{array}{l}\text { H9. La } \\
\text { institucionali } \\
\text { dad se } \\
\text { interrelacion } \\
\text { a con la } \\
\text { transparencia } \\
\text { de la } \\
\text { organización. }\end{array}$ & $\begin{array}{l}\text { Factor de } \\
\text { institucionali } \\
\text { dad }\end{array}$ & $\begin{array}{l}\text { Resultad } \\
\text { o del ITN } \\
\text { entidades } \\
\text { orden } \\
\text { nacional }\end{array}$ & 0,823 & $\begin{array}{l}\text { Correlación } \\
\text { aceptable }\end{array}$ \\
\hline & $\begin{array}{l}\text { H10. El } \\
\text { control- } \\
\text { sanción se } \\
\text { interrelacion } \\
\text { a con la } \\
\text { transparencia } \\
\text { de la } \\
\text { organización. }\end{array}$ & $\begin{array}{l}\text { Factor de } \\
\text { control- } \\
\text { sanción }\end{array}$ & $\begin{array}{l}\text { Resultad } \\
\text { o del ITN } \\
\text { entidades } \\
\text { orden } \\
\text { nacional }\end{array}$ & 0,833 & $\begin{array}{l}\text { Correlación } \\
\text { aceptable }\end{array}$ \\
\hline
\end{tabular}

Fuente: elaboración propia. 
Una vez realizado el cálculo de la correlación entre las variables ISO

9001:2008, ISO 14001:2004, NTC GP 1000:2009, OHSAS 18001:2007 e

ISO 27001:2013 y la variable del resultado del ITN en entidades

nacionales, se puede observar en la tabla 7 que el resultado del coeficiente rho de Spearman oscila entre -0,053 y 0,124, lo que indica la inexistencia de una correlación. En consecuencia, la hipótesis 2, los sistemas de gestión aportan positivamente a la transparencia de la organización, se rechaza debido a la falta de correlación entre las variables.

Tabla 7. Comprobación de hipótesis del modelo conceptual "Los sistemas de gestión afectan la transparencia de la organización"

\begin{tabular}{|c|c|c|c|c|c|}
\hline $\begin{array}{c}\text { Modelo } \\
\text { conceptual }\end{array}$ & Hipótesis & $\begin{array}{l}\text { Variable } \\
1\end{array}$ & $\begin{array}{c}\text { Variable } \\
2\end{array}$ & $\begin{array}{c}\text { Coeficient } \\
\text { e de } \\
\text { correlació } \\
\text { n }\end{array}$ & Resultado \\
\hline \multirow{5}{*}{$\begin{array}{l}\text { Los sistemas } \\
\text { de gestión } \\
\text { afectan la } \\
\text { transparenci } \\
\text { a de la } \\
\text { organización }\end{array}$} & \multirow{5}{*}{$\begin{array}{l}\text { H2. Los } \\
\text { sistemas de } \\
\text { gestión } \\
\text { aportan } \\
\text { positivament } \\
\text { e la } \\
\text { transparenci } \\
\text { a de la } \\
\text { organización } \\
\text {. }\end{array}$} & $\begin{array}{l}\text { ISO } \\
9001: 2008\end{array}$ & \multirow{5}{*}{$\begin{array}{l}\text { Resultado } \\
\text { del ITN en } \\
\text { entidades } \\
\text { del orden } \\
\text { nacional }\end{array}$} & 0,124 & No hay correlación \\
\hline & & $\begin{array}{l}\text { ISO } \\
\text { 14001:200 } \\
4\end{array}$ & & $-0,052$ & $\begin{array}{l}\text { No hay correlación } \\
(-)\end{array}$ \\
\hline & & $\begin{array}{l}\text { NTC GP } \\
1000: 200 \\
9\end{array}$ & & 0,142 & No hay correlación \\
\hline & & $\begin{array}{l}\text { OHSAS } \\
\text { 18001:200 } \\
7\end{array}$ & & $-0,053$ & $\begin{array}{l}\text { No hay correlación } \\
(-)\end{array}$ \\
\hline & & $\begin{array}{l}\text { ISO } \\
27001: 201 \\
3\end{array}$ & & 0,051 & No hay correlación \\
\hline
\end{tabular}

Fuente: elaboración propia.

En síntesis, el desarrollo de este aparte comprueba que la implementación de sistemas de gestión y la aplicación de la normatividad anticorrupción en las entidades no aportan positiva ni negativamente a los resultados del índice de transparencia nacional, puesto que no existe una correlación significativa. 


\section{Limitaciones}

De acuerdo con los resultados obtenidos, la principal limitación de la investigación fue la ausencia de datos y la dificultad para obtenerlos, ya que las páginas web institucionales y las bases de datos del DNP y del DANE no se configuraron como una fuente de información amplia y suficiente para el diligenciamiento de la matriz de caracterización de entidades públicas. Esto imposibilitó su procesamiento en el software estadístico SPSS y obligó al envío de derechos de petición. Sin embargo, estas peticiones fueron respondidas por las entidades públicas extemporáneamente, a pesar de los términos legales, lo que obstaculizó el procesamiento de la información.

\section{Conclusiones}

De acuerdo con la revisión de la normatividad vigente para el periodo 2014-2016, se determina el alto aporte de la normatividad en cuanto a lineamientos, directrices y herramientas que deben implementar las entidades públicas en su quehacer. Sin embargo, el resultado de esta investigación demuestra que no existe incidencia sobre el ITN y sus factores (visibilidad, institucionalidad y control-sanción). Se puede concluir que, aunque las 40 entidades reportaron el cumplimiento de toda la normatividad, es posible que no exista un despliegue adecuado y correcto de la normatividad ni de los sistemas de gestión identificados. Por esto, en el país se siguen materializando hechos de corrupción y no hay disminución significativa del nivel de riesgo.

Ahora bien, como resultado de las caracterizaciones realizadas y la interpretación de las tendencias que se presentan entre los datos procesados en la investigación, se pudo observar que las medidas de tendencia central y de dispersión muestran que los puntajes del ITN de las entidades disminuyeron su dispersión con respecto a la media. Si bien el 
cambio no es significativo, la tendencia de las entidades de la muestra (grupo) percibió un avance de acuerdo con los datos recopilados por la Corporación Transparencia por Colombia. Aunque estas tendencias son positivas y alentadoras, la implementación de sistemas de gestión y la aplicación de la normatividad anticorrupción no inciden en los resultados del ITN.

Así mismo, se puede afirmar que la metodología formulada para la medición del ITN toma en consideración las disposiciones en materia de contratación consignadas en las leyes 80 de 1993 y 1150 de 2007, así como disposiciones en materia de anticorrupción y atención al ciudadano contenidas en las leyes 1474 de 2011 y 1712 de 2014 y el Decreto 19 de 2012, para la creación de indicadores y subindicadores que miden la gestión de las entidades con respecto a estas temáticas. Por lo anterior, la aplicación del mandato de las normas debería incidir positivamente en los resultados del ITN. Sin embargo, en la información procesada en las caracterizaciones de las 40 entidades se observó que, si bien las entidades reportaron para el periodo 2014-2016 que siguieron o implementaron los mandatos de las normas, sus resultados en la medición del ITN en este lapso no fueron ascendentes. Posiblemente, las entidades enfocaron sus esfuerzos en gestionar la contratación y los trámites misionales propios, pero desatendieron las acciones de tratamiento de los riesgos de corrupción que podrían darse en estas operaciones.

De acuerdo con los resultados de la correlación de las variables, se determinó que solo existe correlación entre los factores y el resultado del ITN. Así mismo, no existe una correlación estadística entre la implementación de los sistemas de gestión y la aplicación de la normatividad anticorrupción en el ITN. Se puede concluir que las entidades muestran debilidades en las acciones de 1) divulgación de información y gestión pública, 2) diseño de políticas, metodologías, 
procedimientos y estrategias anticorrupción en diferentes aristas de la gestión pública (planeación, gestión del talento humano, contratación, entre otras), y 3) diseño de tácticas y estrategias que le permitan acercarse al ciudadano, mediante la rendición de cuentas, el control social y el control institucional. Así mismo, se detectan debilidades en la implementación de las estructuras que proponen los sistemas de gestión de calidad, lo que evita que se puedan concentrar en el cumplimiento de los requisitos de ley, los requisitos del ciudadano y el mejoramiento continuo.

La investigación logró responder la pregunta planteada de si los sistemas de gestión y la aplicación de la normatividad anticorrupción han incidido en los resultados del ITN de las entidades públicas del orden nacional con una respuesta negativa. Sin embargo, el desarrollo del primer objetivo específico demuestra que este resultado no se presenta por ausencia de herramientas o lineamientos para que las entidades cumplan con los requisitos evaluados en los factores (visibilidad, institucionalidad y control-sanción), pues se evidencia que la normativa (leyes y decretos) es clara en cuanto a las obligaciones de las entidades frente a temas de anticorrupción y transparencia.

En síntesis, se considera que el cumplimiento a cabalidad de las obligaciones impuestas por la ley por parte de las entidades públicas, así como un compromiso con la implementación de los numerales de las normas técnicas, aumentaría el resultado del ITN y se traduciría en un nivel del riesgo de corrupción más bajo de acuerdo con la metodología planteada por la TpC. 


\section{Referencias}

Corporación Transparencia por Colombia. (2017). Documento metodológico. Indice de transparencia nacional 2015 - 2016.

https://transparenciacolombia.org.co/2017/04/27/documento-metodologico-itn2015-2016/

Departamento Administrativo de la Función Pública. (2015). Guía para la gestión del riesgo de corrupción.

https://www.funcionpublica.gov.co/eva/admon/files/empresas/ZW1wcmVzYV83

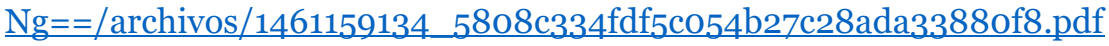

Departamento Nacional de Planeación. (2013, 9 de diciembre). Documento Conpes 167. Estrategia Nacional de la Política Pública Integral Anticorrupción. https://www.funcionpublica.gov.co/eva/gestornormativo/norma.php?i=83097

Hernández-Sampieri, R., Fernández-Collado, C. y Baptista-Lucio, P. (2014). Metodología de la investigación. McGraw Hill.Martínez, C. (2012). Estadística y muestreo. Ecoe Ediciones.

República de Colombia. (2009). Norma Técnica de Calidad en la Gestión Pública - NTC GP 1000:2009. http://www.ibun.unal.edu.co/calidad/docs/normatividad/ntcgp1000.pdf

Spiegel, M. y Stephens, L. (2009). Estadística. McGraw Hill.

Transparencia por Colombia. (2017). Índice de transparencia nacional. Resultados 20152016. https://transparenciacolombia.org.co/2017/03/27/indice-de-transparencianacional-2015-2016/ 